\title{
Nucleon size effects in the parity violating electron scattering asymmetry
}

\author{
J. R. Marinelli ${ }^{1, a}$ and C. A. Graeff ${ }^{2}$ \\ ${ }^{1}$ Departamento de Física, Universidade Federal de Santa Catarina, Florianópolis, Brazil \\ ${ }^{2}$ Departamento de Física, Universidade Tecnológica Federal do Paraná, Campus Pato Branco , Brazil
}

\begin{abstract}
.
We investigate isospin mixing effects in the asymmetry as obtained in parity-violating electron scattering from ${ }^{4} \mathrm{He},{ }^{12} \mathrm{C},{ }^{48} \mathrm{Ca},{ }^{90} \mathrm{Zr}$ and ${ }^{208} \mathrm{~Pb}$ nuclei. The scattering analysis is developed within the distorted wave Born approximation (DWBA) accounting for nucleon form factors. We use a relativistic mean field model, including the $\sigma, \omega, \rho$ and $\delta$ mesons, with density dependent couplings, as well as the electromagnetic interaction . Numerical results are presented for the asymmetry, charge and weak charge radius.
\end{abstract}

\section{Introduction}

Parity violating elastic electron scattering has been an important and probably an unique tool to investigate a series of fundamental properties, which include nuclear structure basic properties as the neutron distribution in nuclei, the strangeness content of the nucleon and the precise determination of the constants that define the Standard Model for the fundamental particles. At this respect, despite the experimental challenge that this type of measurement represents, some data were already obtained for ${ }^{1} \mathrm{H},{ }^{4} \mathrm{He},{ }^{12} \mathrm{C}$ and ${ }^{208} \mathrm{~Pb}$ [1],[2],[3]. New and more precise experimental results are expected in the near future. All those informations are present in the experimental extraction of the so called parity violating (PV) asymmetry, defined as the difference between cross sections with opposite electron polarizations normalized to the their sum, and so a careful and precise knowledge of the nucleon as well as the nucleus structure are needed for the correct interpretation of the measurements. Here we are concerned with the sensitivity of the asymmetry and related observables to the finite size of the nucleon and to the contribution of the anomalous magnetic moment term which is present in the electromagnetic and weak neutral currents of the target, from which the asymmetry is strongly dependent.

\section{Nuclear model and currents}

The nuclear dynamics is described by a relativistic mean field approach. The nucleon model Lagrangian is given as follow:

$$
\mathcal{L}_{i}=\bar{\psi}_{i}\left[\gamma_{\mu} i D^{\mu}-M^{*}\right] \psi_{i}
$$

\footnotetext{
ae-mail: ricardo@fsc.ufsc.br
} 
with

$$
i D^{\mu}=i \partial^{\mu}-\Gamma_{v} V^{\mu}-\frac{\Gamma_{\rho}}{2} \boldsymbol{\tau} \cdot \mathbf{b}^{\mu}-e \frac{1+\tau_{3}}{2} A^{\mu} \quad \text { and } \quad M^{*}=M-\Gamma_{s} \phi-\Gamma_{\delta} \boldsymbol{\tau} \cdot \boldsymbol{\delta} .
$$

To eq. 1 we add the (free) mesonic Lagrangians $\mathcal{L}_{\sigma}, \mathcal{L}_{\omega}, \mathcal{L}_{\rho}$ and $\mathcal{L}_{\delta}$, denoting the contribution of the $\sigma, \omega, \rho$ and $\delta$ mesons respectively, and the eletromagnetic field $\mathcal{L}_{\gamma}$. The couplings $\Gamma$ are density dependents and are parametrized as explained in [5], where we may also find the detailed definition of the whole Lagrangian. The field equations are solved in the Hartree approximation using a previous [4] defined parametrization, which also provides a good description of elastic electron scattering cross sections [6]. For that purpose we must obtain the nuclear charge distribution from the operator [7]:

$$
\hat{J}_{0}^{T}=F_{1}^{T}\left(q^{2}\right) \gamma_{0}+i \frac{\left[\kappa F_{2}^{T}\left(q^{2}\right)\right]}{2 M} \sigma_{0 \nu} q^{v} .
$$

$F_{1}^{T}$ and $F_{2}^{T}$ are the nucleon form factors and we consider here only isoscalar $(T=0)$ and isovector $(T=1)$ components. The weak charge operator is now given in terms of the isospin components as:

$$
\hat{J}_{0}^{W}=\beta_{v}^{0} \hat{J}_{0}^{T=0}+\beta_{v}^{1} \hat{J}_{0}^{T=1} .
$$

The nucleon form factors can now be written in terms of Sachs form factors, for which we choose the parametrization according to reference [8]. Taken the mean value of the above operators with the nuclear wave function derived from the relativistic mean field solution, we obtain both the electromagnetic and weak neutral charge nuclear distribution, in momentum transfer space $q$ :

$$
\begin{gathered}
\varrho_{C}(q)=\frac{G_{D}^{v}(q)}{1+\tau(q)}\left\{\left[\left[1+\tau(q) \mu_{p}\right] \rho_{B}^{p}(q)+\left[\mu_{p}-1\right] \frac{\varrho^{p}(q)}{2 M}\right]\right. \\
\left.+\left[\left[(1-\xi(q)) \mu_{n} \tau(q)\right] \rho_{B}^{n}(q)+\left[(1+\tau(q) \xi(q)) \mu_{n}\right] \frac{\varrho^{n}(q)}{2 M}\right]\right\} F_{C M}(q), \\
\varrho_{W}(q)=\frac{G_{D}^{v}(q)}{1+\tau(q)}\left\{\left[\left[\beta_{v}^{0} A(q)+\beta_{v}^{1} B(q)\right] \rho_{B}^{p}(q)+\left[\beta_{v}^{0} C(q)+\beta_{v}^{1} D(q)\right] \frac{\varrho^{p}(q)}{2 M}\right]\right. \\
\left.+\left[\left[\beta_{v}^{0} A(q)-\beta_{v}^{1} B(q)\right] \rho_{B}^{n}(q)+\left[\beta_{v}^{0} C(q)-\beta_{v}^{1} D(q)\right] \frac{\varrho^{n}(q)}{2 M}\right]\right\} F_{C M}(q),
\end{gathered}
$$

with the following definitions:

$$
\begin{aligned}
& A(q)=1+\left[\left(\mu_{p}+\mu_{n}\right)-\mu_{n} \xi(q)\right] \tau(q) \quad, \quad B(q)=1+\left[\left(\mu_{p}-\mu_{n}\right)+\mu_{n} \xi(q)\right] \tau(q) \\
& C(q)=-1+\left[\left(\mu_{p}+\mu_{n}\right)+\mu_{n} \tau(q) \xi(q)\right] \quad, \quad D(q)=-1+\left[\left(\mu_{p}-\mu_{n}\right)-\mu_{n} \tau(q) \xi(q)\right] \\
& G_{D}^{v}=\left(1+\lambda_{D}^{v} \tau(q)\right)^{-2} \quad, \quad \xi(q)=\left(1+\lambda_{n} \tau(q)\right)^{-1}
\end{aligned}
$$

The constant values are $\lambda_{n}=5.6, \lambda_{D}^{v}=4.97, \mu_{p}=\kappa^{p}+1=2.79, \mu_{n}=\kappa^{n}=-1.91$ and $\tau(q)=\frac{q^{2}}{4 M^{2}}$. The $q$ dependent factor $F_{C M}$ is the usual Tassie center-of-mass correction and the constants $\beta_{v}^{0}$ and $\beta_{v}^{1}$ are as defined in [9]. The functions $\rho_{B}^{p, n}(q)$ and $\varrho^{p, n}(q)$ contain the nuclear structure information and come respectively from the convective and anomalous magnetic moment terms of the operator defined in equation (3). 


\section{Asymmetries, charge and weak radius}

In order to compute the elastic electron scattering cross sections and then the PV asymmetry $\mathcal{A}$ in DWBA, we rewrite the above distributions in coordinate space. All the calculations here are for an electron incident energy of $1063 \mathrm{MeV}$ [3]. For $N=Z$ nuclei, the quantity of interest is the so called isospin mixing factor, defined as

$$
\Gamma(q)=\frac{\mathcal{A}(q)}{\mathcal{A}_{0}(q)}-1
$$

where $\mathcal{A}_{0}(q)$ is the asymmetry calculated assuming perfect isospin symmetry. As discussed elsewhere ([9],[10]), PV electron scattering can be used as a relevant tool to the extraction of Standard Model constants as well as the strangeness content of nuclei. However, isospin mixing can be carefully taken in to account in the process. In fig. 1 we display the isospin mixing calculated for ${ }^{4} \mathrm{He}$ and ${ }^{12} \mathrm{C}$, where we compare the possible size effects in the results. The curves labeled by without nucleon size effects mean that we take $F_{1}=1$ and $F_{2}=0$ in our expressions for the electromagnetic and weak neutral charges.

For $N \neq Z$, the aim is to extract the neutron distribution of the target ([7],[10]). We consider here two cases already studied in the literature: ${ }^{48} \mathrm{Ca}$ and ${ }^{208} \mathrm{~Pb}$ (the target used in the PREX experiment). In those cases, the asymmetry itself is the main observable and are displayed in fig. 2. We have also verified that at the PREX kinematics [3], the asymmetry changes about 3\% when size effects are included, for ${ }^{48} \mathrm{Ca}$, but less then $0.5 \%$ in the lead case. It is also useful to compare the charge $\left(R_{c}\right)$ and weak charge $\left(R_{w}\right)$ radius with and without the nucleon form factor corrections, which are the main observables to be extracted at small angles from elastic electron scattering experiments [11]. In table 1 we show our results, where we have also included figures for ${ }^{90} \mathrm{Zr}$, for which elastic electron scattering [12] and neutron skin measurements [13] were already done. They are defined as:

$$
R_{c}^{2}=\frac{1}{Z} \int d \vec{r} \varrho_{C}(r) r^{2} \quad \text { and } \quad R_{w}^{2}=\frac{1}{\left(\beta_{v}^{0}-\beta_{v}^{1}\right) N+\left(\beta_{v}^{0}+\beta_{v}^{1}\right) Z} \int d \vec{r} \varrho_{W}(r) r^{2}
$$
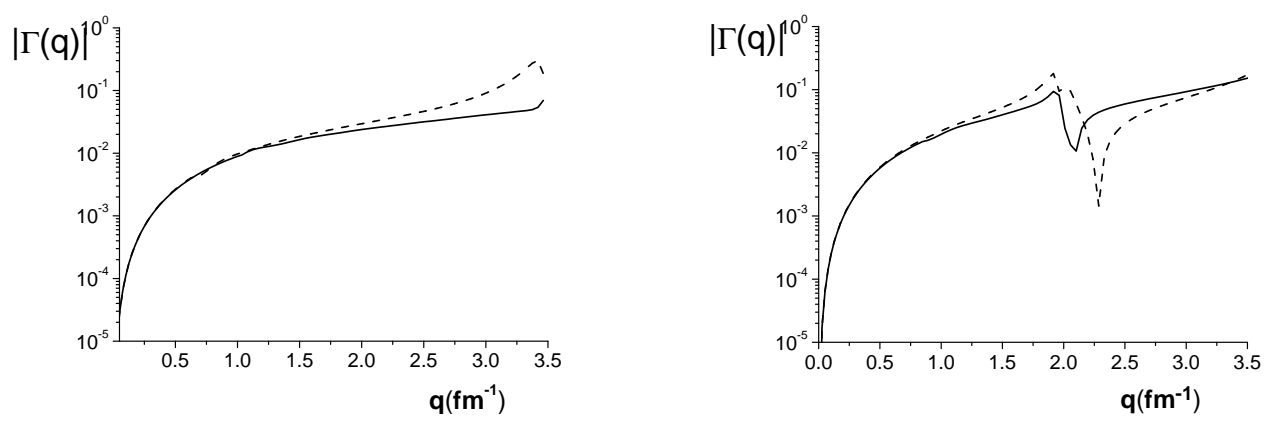

Figure 1. Isospin mixing for ${ }^{4} \mathrm{He}$ (left) and ${ }^{12} \mathrm{C}$ (right) with (full line) and without (dashed line) size effects.

\section{Concluding remarks}

We then conclude that nucleon size effects in PV elastic electron scattering for $N=Z$ nuclei affects very little the isospin mixing function for small $q$ values but can be relevant for medium $q$ experiments, 

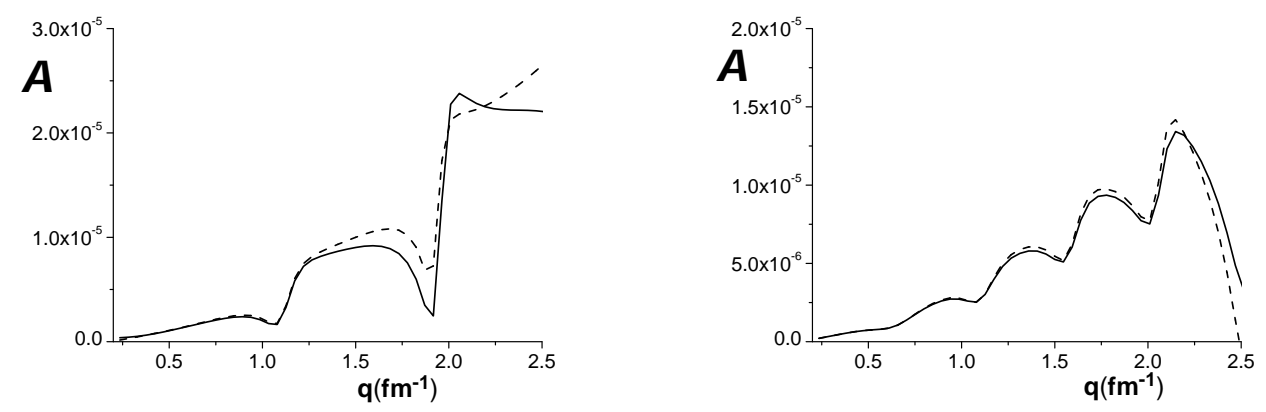

Figure 2. Asymmetry for ${ }^{48} \mathrm{Ca}$ (left) and ${ }^{208} \mathrm{~Pb}$ (right) with (full line) and without (dashed line) size effects.

Table 1. Charge and weak charge radius (in fm), with $\left(R_{c}, R_{w}\right)$ and without $\left(R_{c}^{0}, R_{w}^{0}\right)$ nucleon size effects.

\begin{tabular}{llllll}
\hline Nuclei & $R_{c}$ & $R_{c}^{0}$ & $R_{w}$ & $R_{w}^{0}$ & $R_{c}^{\exp }$ \\
\hline${ }^{48} \mathrm{Ca}$ & 3.442 & 3.410 & 3.669 & 3.591 & 3.474 \\
& & & & & \\
${ }^{90} \mathrm{Zr}$ & 4.259 & 4.224 & 4.367 & 4.299 & 4.269 \\
& & & & & \\
${ }^{208} \mathrm{~Pb}$ & 5.538 & 5.506 & 5.747 & 5.698 & 5.501
\end{tabular}

where the extraction of the strangeness content of the nucleus is aimed. As for highly asymmetric nuclei, where the measurement of the neutron radius is of great interest, we have shown that the effect is less than $1 \%$ for ${ }^{208} \mathrm{~Pb}$, but is bigger for ${ }^{48} \mathrm{Ca}$ (nearly $1 \%$ for the charge and $2 \%$ for the weak charge radius), increasing by $25 \%$ the difference $R_{w}-R_{c}$. For ${ }^{90} \mathrm{Zr}$ the figures are $0.8 \%$ and $1.6 \%$ respectively. We then believe that a case by case analysis should be done for any specific target.

\section{References}

[1] A. Acha et al., Phys. Rev. Lett. 98, 032301 (2007).

[2] K. A. Aniol et al., Phys. Rev. Lett. 96, 022003 (2006).

[3] S. Abrahamyan et al. (PREX Collaboration), Phys. Rev. Lett. 108, 112502 (2012).

[4] C. A. Graeff and J. R. Marinelli, Proceedings of Science, XXXIV Brazilian Workshop on Nuclear Physics,122 (2011), http://pos.sissa.it/cgi-bin/reader/conf.cgi?confid=142.

[5] Avancini, S. S., L. Brito, J. R. Marinelli, D. P. Menezes, M. M. W. de Moraes, C. Providencia and A. M. Santos, Phys. Rev. C79, 035804 (2009).

[6] C.A. Graeff, Phd Thesis, Depto. de Física da Universidade Federal de Santa Catarina, Fpolis, Brazil (2012).

[7] T.W. Donnelly and R.D. Peccei, Phys. Rep. 50, 1 (1979).

[8] S. Galster, H. Klein, J. Moritz, K. H. Schmidt, D. Wegener and J. Bleckwenn, Nucl. Phys. B32, 221 (1971).

[9] O. Moreno, P. Sarriguren, E. Moya de Guerra, J.M. Udias, T.W. Donnelly and I. Sick, Nucl. Phys. A828, 306 (2009).

[10] O. Moreno, P. Sarriguren, E. Moya de Guerra, J.M. Udias, J. Phys. G: Nucl. Part. Phys. 37, 064019 (2010).

[11] C.J. Horowitz and J. Piekarewicz, arXiv:1208.2249v1 [nucl-th] 10 Aug 2012.

[12] L.A. Fajardo, J.R. Ficenec and W.P. Trower, Phys. Letters B37, 363(1971).

[13] K. Yako, H. Sagawa and H. Sakai, arXiv:nucl-ex/0609046v1 29 Sep 2006. 